Mechanisms of Disease

\title{
How receptors work: mechanisms of signal transduction
}

\author{
Edwin R. Chilvers and Tariq Sethi
}

\begin{abstract}
Respiratory Medicine Unit, Department of Medicine (RIE), Rayne Laboratory, Medical School, University of Edinburgh, Teviot Place, Edinburgh EH8 9AG, UK
\end{abstract}

\section{Introduction}

In recent years major advances have been made regarding our understanding of the mechanisms underlying cell surface receptor activation. In particular, how information is transferred from these receptors to the interior of the cell. The aim of this article is to give a brief overview of the major signal transduction pathways employed by cells and to highlight where such information has allowed insight into the molecular mechanisms underlying certain disease states. In many instances this information has permitted the development of novel therapeutic strategies.

While most cells seem to possess a bewildering array of agonist-specific cell surface receptors capable of recognizing incoming hormonal, neurotransmitter, cytokine and growth factor signals, a far more limited number of pathways exist to relay this information to the cell interior. In light of our recent understanding of these signalling pathways, receptors can now be classified in a far more functional way based on the mode of signal transduction rather than purely on the basis of agonist specificity (Figure 1). Such transmembrane signalling mechanisms include receptor regulation of ion channel function, activation of a membrane-associated phospholipase, generation of cyclic nucleotides or stimulation of a receptor-coupled tyrosine kinase. One important caveat to this scheme, however, is the recognition that a single agonist may activate a variey of receptor subtypes (for example, for acetylcholine the $\mathrm{m} 1-\mathrm{m} 5$ muscarinic cholinoceptors), each of which in turn may be coupled to one or more signal transduction pathways.

\section{Receptors that modulate the activity of ion channels}

It has been recognized for a number of years that one of the most efficient ways in which a receptor can alter cell function is by regulating the activity of

Correspondence: E.R. Chilvers, M.R.C.P. (U.K.), Ph.D. Received: 24 June 1994 an ion channel located on the surface of that cell. The presence of ATP-dependent plasmalemmal ion pumps that maintain very high transmembrane concentration gradients for certain ions (for example, sodium, calcium and chloride) permits a rapid flux of such molecules down these gradients when these ion channels are open. In the most simplistic model, best exemplified by either the nicotinic cholinoceptor or the intracellular receptor for the second-messenger inositol 1,4,5-trisphosphate $\left(\operatorname{Ins}(1,4,5) \mathbf{P}_{3}\right)$, the agonist recognition site and ion channel are both contained within a single protein structure. Hence for the nicotinic cholinoceptor, the binding of a single molecule of acetylcholine to one of the $\alpha$-subunits of the receptor results in a conformational change of the intrinsic ion channel component of the protein. This in turn permits the transient influx of sodium into the cell causing membrane depolarization and the generation of an action potential.

The inhibitory neurotransmitters, gamma aminobutyric acid (GABA) and glycine, likewise bind to receptors that possess intrinsic ion channel activity. When these channels open, chloride ions flow into the cell, which has the effect of increasing membrane potential and hence reducing the likelihood of neuronal activation. Attempts have been made to exploit this ability of GABA to inhibit neuronal activation by the development of receptor

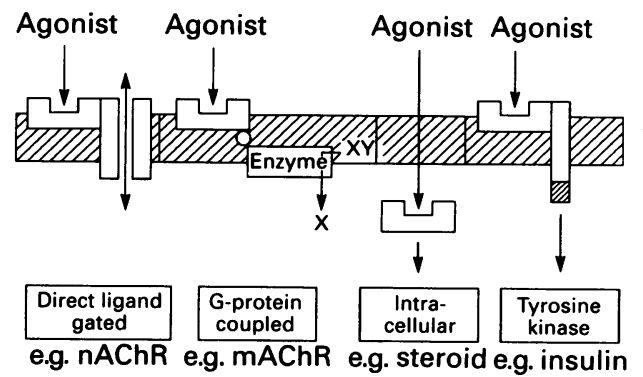

Figure 1 Functional classification of cell surface receptors. $\mathrm{nAChR}=$ nicotinic acetylcholine receptor; $\mathrm{mAChR}$ $=$ muscarinic acetylcholine receptor. 
agonists such as muscimol. It is also well recognized that many of the sedative and anxiolytic effects of the benzodiazepines relate to their ability to potentiate the effects of GABA by binding to an accessory site present on the $\mathrm{GABA}_{\mathrm{A}}$ receptor. It is of interest that the recent cloning of the nicotinic cholinoceptor, GABA and glycine receptors has revealed striking sequence homology suggesting that they are all members of a single family of receptor proteins that have evolved from a common ancestor.

A variety of other mechanisms exist to control ion channels that lack such intrinsic agonist binding receptor sites. For example, $\mathrm{N}$-, T- and L-type plasmalemmal calcium channels can be activated by sensing and responding to changes in membrane polarization. Opening of these channels results in calcium influx followed by activation of a family of intracellular $\mathrm{Ca}^{2+} /$ calmodulin-dependent protein kinases. These channels, which are particularly important in regulating vascular smooth muscle tone, represent the major site of action of the so-called ' $\mathrm{Ca}^{2+}$ channel antagonists'. Cell surface receptors can also be linked to ion channels either via an intermediate guanine nucleotide binding protein or G-protein (for example, the acetylcholine-regulated cardiac $\mathrm{K}^{+}$channel), or by the generation of a diffusable intracellular second messenger. Other ion channels appear to be regulated by alterations in cellular adenosine triphosphate (ATP) or $\mathrm{Ca}^{2+}$ concentration. These include the recently discovered set of ATP- and $\mathrm{Ca}^{2+}$-regulated $\mathrm{K}^{+}$channels, which mediate $\mathrm{K}^{+}$ efflux and hence hyperpolarization of the plasma membrane which attenuates $\mathrm{Ca}^{2+}$ entry and hence inhibits cell activation. Knowledge of this latter group of channels has revealed the molecular basis for the hypoglycaemic effect of glibenclamide, which appears to be mediated by its ability to block $\mathrm{K}^{+}{ }_{\text {ATP }}$ channels. In view of the membrane hyperpolarization properties of these channels, a number of $\mathrm{K}^{+}$-channel agonists are now being evaluated for use in asthma and hypertension.

\section{Receptors that activate membrane associated phospholipases}

While historically the membrane phospholipids have been regarded as fulfilling a largely structural role in maintaining an environment in which receptors and ion channels can operate, it is apparent that these molecules can themselves serve an important role in signal transduction. In particular, the superfamily of G-protein-linked receptors containing seven membrane spanning domains are known to activate one or more membrane associated phospholipase enzymes. This results in the generation of a series of phospholipid-derived, intracellular second-messenger molecules. The site at which these individual enzymes, namely phos- $\frac{\mathbb{Q}}{\circ}$ pholipase C, D and $A_{2}$ (PLC, PLD and PLA P $_{C}$. hydrolyse membrane phospholipids is depicted in $\Rightarrow$ Figure 2. It should be noted that while each of these $\stackrel{9}{\stackrel{9}{9}}$ enzymes appear to be linked preferentially to the hydrolysis of a particular class of phospholipid (for 흘 example, PLC mediating the cleavage of phos- $\frac{\sigma}{\sigma}$ phatidylinositol 4,5-bisphosphate), there are $\mathbb{Q}$

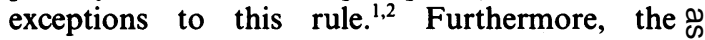
identification of multiple, functionally distinct, $\vec{O}$ PLC and PLA ${ }_{2}$ isoenzymes, which display differential tissue distribution, has opened up the prospect that these could be valuable targets for the development of new drugs.

One of the best understood pathways in this group is that involving receptor-mediated PLC $\circlearrowleft$ activation, which results in the generation of the second-messengers Ins $(1,4,5) \mathrm{P}_{3}$ and diacylglycerol 0 (DAG) (Figure 3a). ${ }^{3,4}$ While the water-soluble $\stackrel{\infty}{\omega}$ Ins $(1,4,5) \mathbf{P}_{3}$ falls into the cytoplasm to bind to its $\omega$ own intracellular receptor to initiate $\mathrm{Ca}^{2+}$ release $ᄋ$ from internal stores, the DAG moiety is retained in the membrane and facilitates the activation of one or more of a family of protein kinase $\mathrm{C}$ molecules. ${ }^{5} \stackrel{\Phi}{ }$ Not only has the elucidation of this pathway been 3 vital to our understanding of how plasma mem- $\mathbb{D}$ brane-bound receptors regulate $\mathrm{Ca}^{2+}$ flux insige cells, it has also led to the development of a number of PLC inhibitors, including flosequinan, that af proving to be effective vasodilator agents. Although the side-effect profile of flosequinan has led to its withdrawal from clinical use, it is likely that alternative PLC inhibitors will be developed in the near future. An understanding of the PLC/ $\triangle$ Ins $(1,4,5)_{3} /$ DAG pathway has also provided some $\overrightarrow{\overrightarrow{0}}$ insight into the action of lithium in manic depression, which is thought to dampen neuronal activity by inhibiting the inositol monophosphatase enzyme, which results in reduced inositol recycling and phosphatidylinositol 4,5-bisphosphate resynthesis and hence decreased $\operatorname{Ins}(1,4,5) \mathrm{P}_{3}$ production

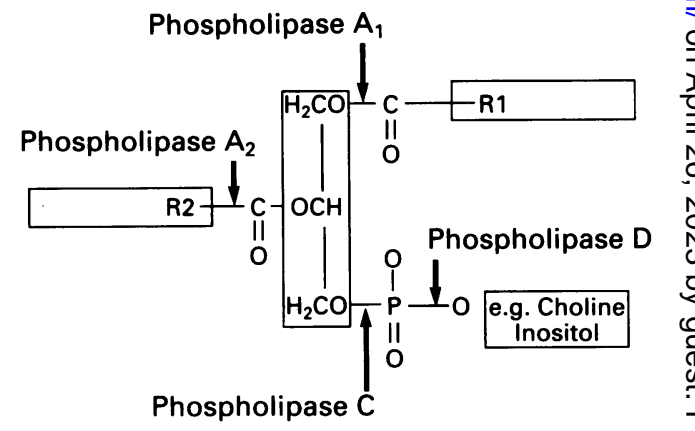

Figure 2 Sites of phospholipid hydrolysis by the major phospholipases. 

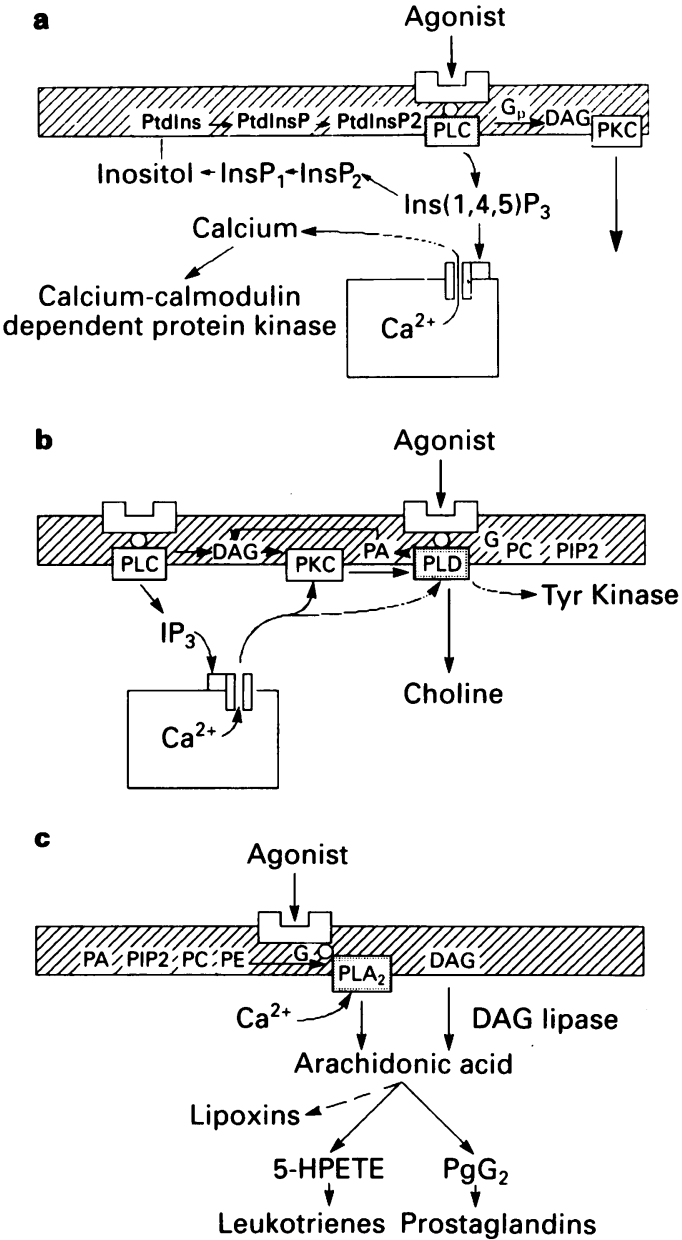

Figure 3 Signal transduction pathways involving PLC, PLD, and $P_{2} A_{2} . P A=$ phosphatidic acid; $P C=$ phosphatidylcholine; $\mathrm{PE}=$ phosphatidylethanolamine; PtdIns $=$ phosphatidyinositol; $\mathbf{G}=$ G-protein; DAG $=$ diacylglycerol; $\mathrm{IP}_{3}=$ inositol triphosphate.

(see Figure 3a). Furthermore, the congenital absence of one of the major enzymes involved in the metabolism of Ins $(1,4,5) \mathrm{P}_{3}$, namely the Ins $(1,4,5) \mathrm{P}_{3}$ 5-phosphatase, is now recognized to be the molecular defect underlying Lowe's occulocerebrorenal syndrome. ${ }^{6}$

Guanine nucleotide-binding proteins (G-Proteins), which consist of a family of highly homologous, heterotrimeric ( $\alpha, \beta$ - and $\gamma$-subunits) guanine triphosphate (GTP)-binding proteins, play a pivotal role in the transfer of information between the receptor and these membrane phospholipases. This is true not only for receptormediated phospholipase activation, but relates also to the linkage of receptors to ion channels and such enzymes as adenylyl and guanylyl cyclase (see below). Activation of a G-protein by a receptor results in GTP displacing GDP on the G-protein $\alpha$-subunit, which in turn leads to subunit dissociation and the generation of $\alpha$-GTP and free $\beta \gamma$ subunits. ${ }^{7}$ While traditionally the $\alpha$-GTP subunit has been considered to be the active product of this reaction, with differences in $\alpha$-subunit structure determining in large part the downstream specificity of the G-protein, ${ }^{8}$ it is now recognized that the liberated $\beta \gamma$ complex also plays an important signalling role, for example, in the activation of the $\beta 2$ isoform of PLC. ${ }^{9}$ The importance of G-proteins in signal transduction has been highlighted by the observation that certain G-proteins, notably Gs $\alpha$, which stimulated adenylyl cyclase, and Gt $\alpha$ (transducin), which activates a retinal cyclic GMPdependent phosphodiesterase, can be inactivated by pertussis toxin. Consequently, many of the clinical manifestations of whooping cough, including the side effects observed following vaccination, are thought to relate to the ability of this toxin to impede transmembrane signalling by causing adenosine diphosphate (ADP)-ribosylation of these G-proteins. In addition, certain G-protein mutants have been identified in tumours such as the GH-secreting pituitary adenoma, which results in unrestrained G-protein activation. ${ }^{10}$ Furthermore, the uncoupling of certain receptors from their target G-proteins has been proposed as one of the main mechanisms underlying the anti-tumour effect of agents such as [D-Arg ${ }^{1}, \mathrm{D}-\mathrm{Arg}^{1}, \mathrm{D}-\mathrm{Phe}^{5}$, D-Trp ${ }^{7,9}$, Leu ${ }^{11}$ substance $P$, which are being evaluated for their use in treating small cell lung cancer. ${ }^{11}$

\section{Receptors linked to cyclic nucleotide generation}

One of the earliest groups of second messengers identified in cells were the cyclic nucleotides cAMP and cGMP. These molecules mediate their effect by regulating the activity of their respective cyclic nucleotide-dependent protein kinases (PKA and PKG). ${ }^{12}$ Although the basic mechanisms underlying the activation of the enzymes involved in the formation of these molecules, namely adenylyl and guanylyl cyclase, is very similar to that outlined for the membrane phospholipases, two important differences exist. Firstly, for adenylyl cyclase, the activity of this enzyme appears to be regulated not only by receptor $(\mathrm{G} \alpha \mathrm{s})$-mediated stimulation but also by receptor-mediated inhibition via $G \alpha i$ (Figure 4a). Secondly, a discrete group of nonmembrane bound guanylyl cyclases exist that mediate the effects of nitric oxide and represent the target for the nitrovasodilators such as sodium nitroprusside (Figure 4b). An understanding of the molecular diversity that exists in the family of phosphodiesterase enzymes responsible for the 


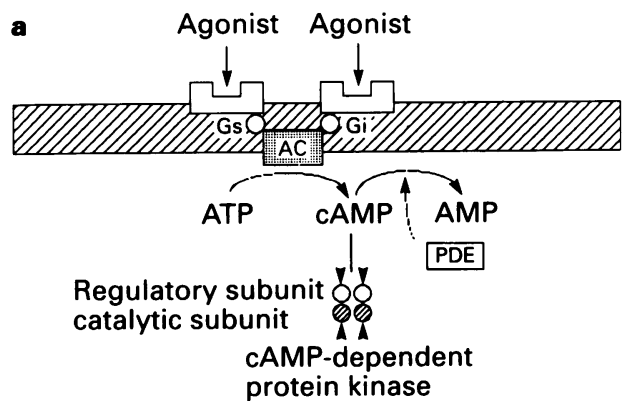

b

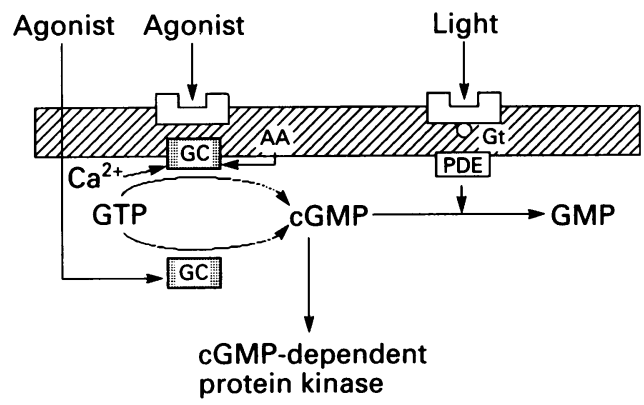

Figure 4 Regulation of cAMP and cGMP in cells. $\mathbf{G}=\mathrm{G}$-protein; $\mathbf{A T P}=$ adenosine triphosphate; cAMP = cyclic adenosine monophosphate; $\mathrm{PDE}=$ phosphodiesterase; $\mathrm{AC}=$ adenylyl cyclase; $\mathrm{GC}=$ guanylyl cyclase; $\mathbf{A A}=$ arachidonic acid; $\mathbf{G T P}=$ guanosine triphosphate; cGMP = cyclic guanosine monophosphate.

breakdown of cGMP and cAMP, and the differences in the cellular distribution of these enzymes, has led to the development of a wide range of selective inhibitors that are being evaluated in the management of patients with, for example, heart failure refractory to more conventional therapy.

\section{Receptors with intrinsic tyrosine kinase activity}

The final major signal transduction pathway that exists in cells relates to the family of receptors that contain intrinsic tyrosine kinase activity (Figure 5). This includes the majority of the growth and colony stimulation factors, for example, plateletderived growth factor, epidermal growth factor and the granulocyte colony-stimulating factors, GM-CSF and G-CSF. The central feature of this pathway is that receptor activation results in phosphorylation and activation of a tyrosine kinase domain located on the cytosplasmic tail of these receptors which activates a set of 'tyrosine kinase-associated proteins'. While the downstream consequences of such interactions are highly diverse and complex, some of the most important
Agonist Agonist

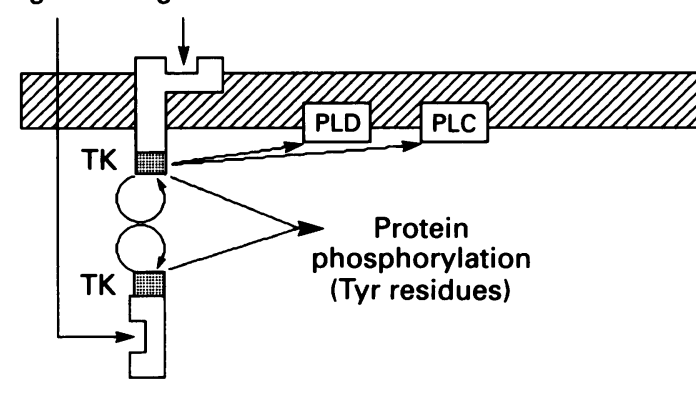

Figure 5 Agonist-stimulated tyrosine kinase activation

and best characterized targets for this pathway are PLC $\gamma,{ }^{13}$ ras-GAP, mitogen-activated protein (MAP)-kinase and the phosphoinositide $3-\mathrm{OH}$ kinase. ${ }^{14}$ Some of the difficulties encountered in dissecting the precise details of the above pathway relate to the observation that a number of $G$ protein-linked receptors (such as bombesin) can in addition activate a set of (cytosolic) protein tyrosine kinases (for example, focal adhesion kinase, $\mathrm{pp} 125^{\mathrm{FAK}}$ ), and that protein kinase $\mathrm{C}$ (PKC), $\mathrm{Ca}^{2+}$ and arachidonic acid likewise can converge on this pathway. Nevertheless, this patho way clearly plays a pivotal role in mediating the effects of many of the growth and cellula differentiation factors, and its close association with certain non-receptor oncogene products has stimulated intense research in this area with the particular aim of developing new anti-tumour agents.

The only major set of receptors not covered by the above classification system are those that bind glucocorticoids and thyroxine. This reflects the ability of this class of agents to access the interior of the cell directly and not be confined to the extracellular space. As a consequence, the glucocorticoid receptor, for example, is located within the cytoplasm of cells and has a discrete mode of action compared to receptors located on the plasma membrane. Under basal conditions the glucocorticoid receptor consists of two steroid binding sites each complexed with a heat-shock protein (HSP90) which dissociate following activation. This permits transfer of the glucocorticoid receptor to the nucleus where it interacts with a glucocorticoid response element (GRE) to modulate gene transcription.

With regard to the overall regulation of a cell's response to what is often a complex set of incoming signals, it is important to understand that there exist a number of routes permitting the above signal transduction pathways to interact. This so-called 'cross-talk' probably has a major role to play in fine tuning the cell's overall response to 
agonist stimulation. A pertinent example of this includes the ability of PKC to phosphorylate and uncouple cell surface receptors and to activate the Ins $(1,4,5) \mathbf{P}_{3}$-phosphatase. ${ }^{15}$ Additional examples include the ability of $\mathrm{Ca}^{2+}$ to augment receptormediated PLC activation and activate the Ins $(1,4,5) P_{3}$ 3-kinase responsible for the formation of Ins $(1,3,4,5) P_{4},{ }^{16}$ and for protein kinase $A$ to phosphorylate $P L C \gamma^{17}$ and the Ins $(1,4,5) P_{3}$ receptor. In addition, $\mathrm{PKC}$ appears to play a vital role in protecting cells from undergoing apoptosis (programmed cell death) in response to sustained agonist-stimulated increases in $\mathrm{Ca}^{2+}$ or cAMP. ${ }^{18}$

\section{References}

1. Exton, J.H. Signalling through phosphatidylcholine breakdown. J Biol Chem 1990, 265: 1-4.

2. Thompson, N.T., Bonser, R.W. \& Garland, L.G. Receptorcoupled phospholipase D and its inhibition. TIPS 1991, 12: 404-408.

3. Berridge, M.J. \& Irvine, R.F. Inositol phosphates and cell signalling. Nature 1989, 341: 197-205.

4. Cockcroft, S. \& Thomas, G.M.H. Inositol-lipid-specific phospholipase $\mathrm{C}$ isoenzymes and their differential regulation by receptors. Biochem J 1992, 288: 1-14.

5. Hug, H. \& Sarre, T.F. Protein kinase C isoenzymes: divergence in signal transduction? Biochem $J$ 1993, 291: $329-343$.

6. Attree, O., Olivas, I.M., Okabel, O., Bailey, L.C., Nelson, D.L., Lewis, R.A., McInnes, R.R. \& Nussbaum, R. The Lowe's occulocerebrorenal syndrome gene encodes a protein highly homologous to inositol polyphosphate-5-phosphatase. Nature 1992, 358: 239-242.

7. Taylor, C.W. The role of $\mathbf{G}$ proteins in transmembrane signalling. Biochem $J$ 1990, 272: 1-13.

8. Neer, E.J. \& Clapham, D.E. Roles of G protein subunits in transmembrane signalling. Nature 1988, 333: 129-134.

9. Katz, A., Wu, D. \& Simon, M.I. Subunits $\beta \gamma$ of heterotrimeric $G$ protein activate $\beta_{2}$ isoform of phospholipase C. Nature 1992, 360: 686-689.

10. Landis, C.A., Masters, S. B., Spada, A., Pace, A.M., Bourne, H.R. \& Vallar, L. GTPase inhibiting mutations activate the alpha chain of $\mathrm{G}(\mathrm{s})$ and stimulate adenylyl cyclase in human pituitary tumours. Nature 1989, 340: 692-696.
Recent research therefore has shed considerable light on the ways in which cells transfer information from their surface receptors to the interior of the cell. This knowledge has allowed a far more detailed understanding of how certain drugs work and has provided several unique opportunities to develop novel therapeutic agents to target these signalling pathways. The identification of multiple and often tissue-specific isoforms of many of the enzymes involved in these signalling cascades has increased the likelihood that such a strategy will continue to reap rewards.

11. Sethi, T., Langdon, S., Smyth, J. \& Rozengurt, E. Growth of small cell lung cancer cells: stimulation by multiple neuropeptides and inhibition by broad spectrum antagonists in vitro and in vivo. Cancer Res 1992, 52: 2737s-2742s.

12. Takemoto, D.J., Gonzalez, K., Udovichenko, I. \& Cunnick, J. Cyclic GMP-regulated cyclic nucleotide phosphodiesterases. Cell Signalling 1993, 5: 549-556.

13. Rhee, S.G. Inositol phospholipid-specific phospholipase C: interaction of the $\gamma_{1}$ isoform with tyrosine kinase. TIBS 1991, 16: $297-301$.

14. Stephens, L.R., Jackson, T.R. \& Hawkins, P.T. Agoniststimulated synthesis of phosphatidylinositol $(3,4,5)$ triphosphate: a new intracellular signalling system? Biochim Biophys Acta 1993, 1179: 27-75.

15. Connolly, T.M., Lawling, W.J. \& Majerus, P.W. Protein kinase $C$ phosphorylates human platelet inositol trisphosphate 5-phosphomonoesterase, increasing the phosphatase activity. Cell 1986, 46: 951-958.

16. Biden, T.J. \& Wollheim, C.B. $\mathrm{Ca}^{2+}$ regulates the inositol tris/tetrakisphosphate pathway in intact and broken preparations of insulin-secreting RINm5F cells. J Biol Chem 1986, 261: $11931-11934$.

17. Kim, U.-H., Kim, J.W. \& Rhee, S.G. Phosphorylation of phospholipase $\mathrm{C}$-gamma by cAMP-dependent protein kinase. J Biol Chem 1989, 264: 20167-20170.

18. McConkey, D.J., Orrenius, S. \& Jondal, M. Cellular signalling in programmed cell death (apoptosis). Immunol Today 1990, 11: $120-121$. 